\title{
Looking for long-range correlations among the EEE telescopes
}

\section{P. La Rocca ${ }^{a, b, *}$ on behalf of the EEE Collaboration}

(a complete list of authors can be found at the end of the proceedings)

${ }^{a}$ Dipartimento di Fisica e Astronomia, Università di Catania, Catania, Italy

${ }^{b}$ INFN Sezione di Catania, Catania, Italy

E-mail: paola.larocca@ct.infn.it

The search for long-range correlations among air showers is one of the main goal of the Extreme Energy Events (EEE) Project. The existence of such events has only been supposed theoretically through several physical mechanisms, the most convincing being the so-called GZ effect, based on the photodisintegration of a heavy primary nucleus in the solar field. Even with a large detector coverage, current rate expectations are of few events per year.

To measure time correlations among distant air showers, sparse arrays of detection stations spread over large areas are needed. A very limited number of experimental setups can perform this measurement and few experimental results have been reported over the past years.

Started in 2004 the EEE project is a network of about 60 cosmic muons tracking telescopes made by 3 wide area Multigap Resistive Plate Chambers (MRPCs), sensitive to the direction of incident charged cosmic particles. The telescopes are distributed over the whole Italian territory, thus making the EEE array an ideal tool for the detection of long-range time correlations among extensive air showers.

In this paper we will describe the analysis strategies adopted to search for such rare correlation events, together with the results obtained analysing the full statistics collected by the EEE telescopes.

$37^{\text {th }}$ International Cosmic Ray Conference (ICRC 2021)

July 12th - 23rd, 2021

Online - Berlin, Germany

\footnotetext{
${ }^{*}$ Presenter
} 


\section{Physics motivations}

Since their discovery, cosmic rays represent one of the most fascinating field of research because of their unique characteristics. One way of learning about cosmic rays is using detectors on Earth's surface to observe aspects of the Extensive Air Showers (EASs) produced by primary cosmic rays impacting our atmosphere. The study of longitudinal and lateral profiles of EASs provides an indirect information about the energy, direction and composition of primary cosmic rays generating them. The observatories currently in operation for the study of cosmic rays usually consist of arrays of several detectors covering an area large enough to detect EASs induced by extremely high-energy cosmic rays. However, they are usually not able to observe the arrival of widely-separated simultaneous extensive air showers. In this sense, a globally-distributed network of detectors would be an innovative instrument for the investigation of cosmic rays, providing the possibility to reveal phenomena not yet experimentally observed on a planetary scale.

The mechanisms which are able to explain the existence of correlations between individual cosmic ray showers at large distances are still not known, representing an interesting challenge in cosmic ray physics. Basically, they may be classified into two different classes: one implying the existence of two primary cosmics, originating from the same source and producing independent showers in the Earth's atmosphere; the other related to the possibility that a single primary interacts with the interstellar medium and/or the radiation field, thus producing two intermediate products which in turn produce the two showers. The second category is based on the assumption that the space through which cosmics travel before interacting with the atmosphere is not an empty space; on the contrary it is an extremely complex environment, where different processes may occur. A leading example is the Gerasimova-Zatsepin (GZ) effect [1], which predicts the possibility to observe correlation of individual cosmic showers at large distances - in the order of ten or hundred $\mathrm{km}$ resulting from the photodisintegration of ultra high-energy cosmic ray nuclei in the solar radiation field. The distance among the showers, the influence of the Sun magnetic field and the probability to observe such events depend however upon several factors. Detailed numerical simulations (see for example Refs. [2-10]) have been carried out in the past years but the existing estimates of the event rate are subject to large uncertainties mainly because the nuclear composition of ultra high-energy cosmic rays is poorly known: current rate expectations range from $10^{-3}$ to 1 event per $\mathrm{km}^{2}$ per year. Moreover, the GZ effect is just one of the phenomena which can explain the observation of simultaneous widely-separated showers. As a consequence, the true rate of long-range coincidences between EASs is essentially unknown.

To date, no certain evidence of these mechanisms has been experimentally confirmed, possibly owing to the limitations of existing observational technology. In the past, the LAAS [11-13] and ALTA/CZELTA $[14,15]$ Collaborations have reported on preliminary large-scale coincidences between extensive air showers, whereas other detector networks, employing a large variety of technologies, are currently performing campaigns to detect simultaneous showers. In this scenario the EEE Project [16] has built a sparse array of cosmic ray telescopes, spread over the Italian territory, with the ambitious goal of looking for long-range correlations between EASs.

In Section 2 a detailed description of the EEE experiment and its detection setup is given, while the analysis strategy and the first preliminary results are addressed in Section 3 and 4 respectively. 


\section{The EEE experiment}

The EEE experiment was born from an initiative of the Enrico Fermi Research Center in Rome, in collaboration with INFN (Italian National Institute for Nuclear Physics), CERN and MIUR (the Italian Ministry of Education, University and Research). The EEE experiment design features an array of charged-particle tracking telescopes, based on MRPC technology, which are very similar, conceptually, to the chambers developed for the Time-Of-Flight system of the ALICE experiment at LHC. Currently, with about 60 telescopes installed across Italy and CERN, the EEE network is the largest and longest-living MRPC-based system. Except for few cases, where telescopes are installed in the same metropolitan area, the separation distance between telescopes pairs exceeds the size of individual EASs, thus allowing to search for potential rare coincidences between two different air showers detected in sites placed hundreds of kilometres apart. Moreover, thanks to such a configuration, the research program of the EEE experiment also includes the study of the properties of the local muon flux measured by each telescope and the detection of high-energy extensive air showers by looking at the coincidences between telescopes in the same metropolitan area.

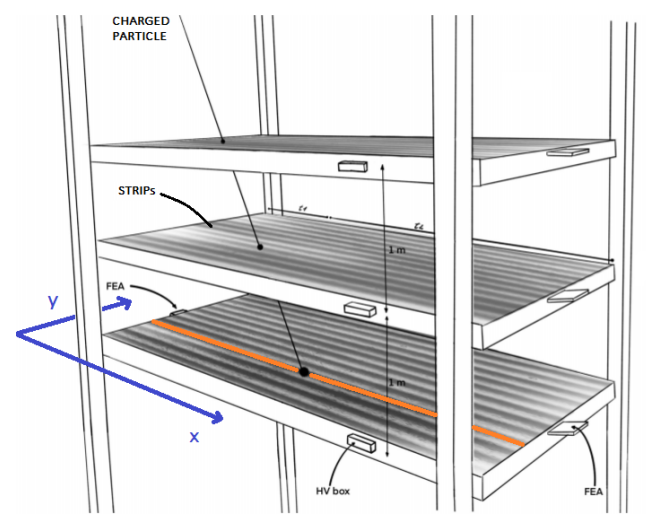

Figure 1: Sketch of a EEE telescope.

A typical EEE telescope, sketched in Fig.1, consists of 3 large area MRPCs, that have been specifically designed in order to fulfil several requirements: large detection area $\left(\sim 80 \times 160 \mathrm{~cm}^{2}\right)$, contained cost, long term operation, high efficiency and good time resolution. Each MRPC has six gaps obtained by a sandwich of 7 glass plates spaced $300 \mu \mathrm{m}$, flushed with a small but continuous flow of gas mixture made of $\mathrm{C}_{2} \mathrm{H}_{2} \mathrm{~F}_{4}(98 \%)$ and $\mathrm{SF}_{6}(2 \%)$. The high voltage applied to the outer glasses, typically in the range $18-20 \mathrm{kV}$, is provided by a set of DC/DC converters and generates a uniform electric field between glass electrodes. The ionization signal produced by a charged particle traversing the detector is induced on the 24 readout copper strips $(2.5 \mathrm{~cm}$ wide with 0.7 $\mathrm{cm}$ pitch), that are laid out on both sides of the stack. The signal drifts to both the ends of the strip and is read by front-end cards: the $y$ coordinate (short side) is determined by the strip on which the signal is induced, whereas the time difference of the signals arriving at the two strip ends, measured by commercial multi-hit TDCs, provides the $x$ coordinate (long side). The impact position is reconstructed on each chamber with a resolution of the order of $1 \mathrm{~cm}$ on the transverse coordinate (as expected due to the strip pitch) and $1.5 \mathrm{~cm}$ on the longitudinal coordinate, in very good 
agreement with the expectations. The average time resolution is approximately $240 \mathrm{ps}$, allowing a precise measurement of the time of flight between the top and bottom chamber. An efficiency better than $90 \%$ is reached by $77 \%$ of the chambers, with possible sources of inefficiencies related to ageing effects or/and dead strips $[17,18]$. The trajectory of the charged particles crossing a telescope is obtained by fitting the impact points reconstructed on the 3 MRPCs, which are placed at a distance of $50 \mathrm{~cm}$ from each other. Each telescope is equipped with a Spectracom PCI Global Positioning System (GPS) card, that provides the event time stamp with a precision of the order of $40 \mathrm{~ns}$, guaranteeing the synchronization between the telescopes.

The EEE network has grown over the years, starting its operation in 2004 with a set of only 7 pilot sites and now running at full operation with up to 62 telescopes in simultaneous and centralized data taking. The detector performance and the statistics collected so far by the EEE telescopes allow a large variety of studies, which include for example the measure of the local cosmic ray flux and its space weather-correlated features [19, 20], the search for anisotropies in the muon angular distribution [21] and the detection of extensive air showers producing time correlations in EEE stations in the same metropolitan area [22]. In addition to these studies, the extension of the EEE network opens the possibility to measure time correlated events at distances never addressed before. The separation distances between all possible pairs of EEE telescopes is reported in Figure 2, showing that the largest distance between the telescopes of the EEE array is about $1200 \mathrm{~km}$ (between CERN and Catania).

Finally, it must be mentioned that the peculiarity of the EEE Project is its innovative outreach approach: almost all the telescopes are installed in Italian high-schools, where young students and teachers play a primary role in the experiment, actively participating to the construction, operation, data taking and maintenance of their detector [23].

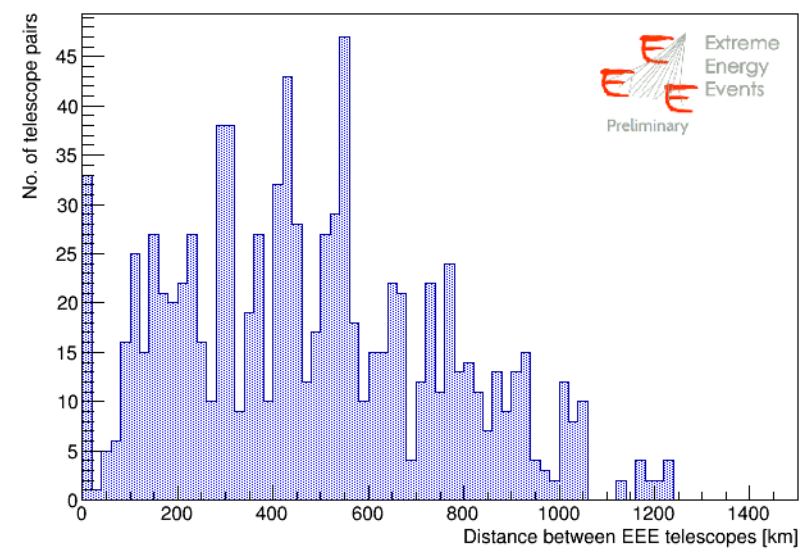

Figure 2: Distribution of the distances between any telescope pair in the EEE array, arranged with a bin size of $20 \mathrm{~km}$.

\section{Analysis strategy}

The main challenge in the search of long-range correlations between distant air showers is related to their rare occurrence, which makes them easily confused with spurious coincidences. As 
a consequence, different strategies have been adopted, aiming at selecting a pure sample of EAS events and thus reducing the huge amount of spurious coincidence events between telescopes. A first attempt was performed limiting the study of time correlations to those sites of the network that host at least two close telescopes (at a distance of few $\mathrm{km}$ ): in these sites (in total 10), EAS events are selected requiring the coincidence between close telescopes; once EASs are selected, we looked for possible time correlations among these sites, for an equivalent data taking time of more than 4000 days. Even if such strategy led to the observation of 5 candidate events of EAS correlations with unusually low p-value [24, 25], the analysed data correspond to a small fraction of the whole statistics available, since most of the telescopes were not included in the analysis.

Alternatively, thanks to the large detection area and acceptance of the EEE telescopes, sometimes two or more cosmic particles can be simultaneously detected by single telescopes in the same event. Such a circumstance can be related to secondary cosmics belonging to the same EAS or to particles originated from interactions of cosmic rays in the building surrounding the detector. Regardless of their origin, the selection of multi-track events allows the reduction of the spurious coincidence level when looking for time correlations between telescope pairs. As deduced from the histogram in Figure 3, which shows a typical distribution of the number of tracks per event in one EEE telescope (CERN-01), the number of events with 2 or more tracks corresponds to a few percent of the total number of events. As a consequence, if the average acquisition rate of each telescope ranges from 15 to $50 \mathrm{~Hz}$, depending on several factors (like the altitude and the detector plane distance), the multi-track count rate is of the order of $10^{-2} \mathrm{~Hz}$ if $N_{\text {tracks }}>3$. This leads to a level of spurious coincidences of the order of few events per year in a time window of $1 \mathrm{~ms}$, that is low enough if compared to the probability to observe rare coincidences between EASs.

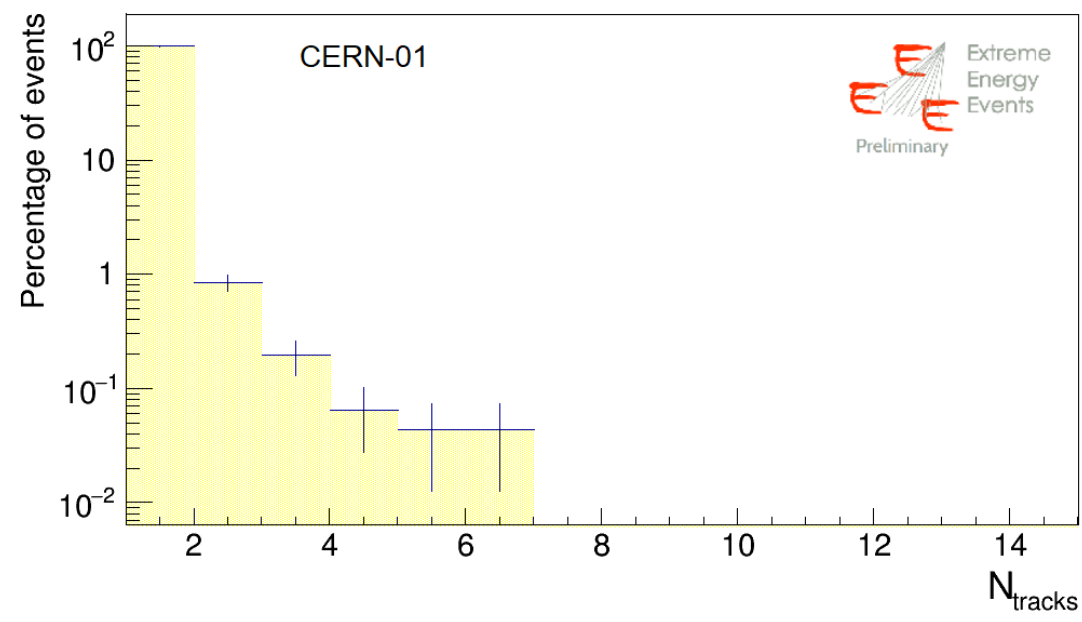

Figure 3: Distribution of the number of tracks per event, reported as a percentage of the total number of events.

All the statistics collected during the coordinated data-taking period (2015-2020) were processed at CNAF in order to pre-select coincident multi-track events among all possible pairs of EEE telescopes within $\mathrm{a} \pm 2 \mathrm{~s}$ time window. At this stage basic quality cuts were applied: the tracks reconstructed in each event must have a $\chi^{2}<10$ and be approximately parallel to the seed track (i.e. 
the track with the best $\chi^{2}$ in the event), requiring that the cosine of the relative angle between each track and the seed track is greater than 0.8. Under these conditions a sample of about 25 million of coincidences was selected, almost all being spurious events due to the large coincidence time window used. An additional cut on the separation distance among the involved sites $(\mathrm{d}>5 \mathrm{~km})$ was applied in order to exclude coincidences between telescopes placed in the same town, which are most likely due to the detection of a single EAS by two close telescopes.

The analysis strategy is based on the comparison of the experimental coincidences observed in a small time window with respect to the expected background of spurious coincidences. An excess of events could indicate the possible existence of long-range correlations between EASs. Such comparison was performed for different values of time window, lower than $5 \mathrm{~ms}$ (i.e. the light-time corresponding to the largest distance covered by the telescope pairs of the EEE array), and investigating different conditions on the number of tracks per event, on the distance between the sites, on the $\chi^{2}$ of the tracks and on the parallelism of the tracks in each telescope.

\section{Results and outlook}

The optimization of the cuts discussed in the previous Section led to the result shown in Figure 4, which was obtained imposing the conditions $\mathrm{d}>5 \mathrm{~km}$ (to exclude true coincidences produced by two telescopes detecting the same EAS) and $N_{\text {tracks }}>3$. In the plot the number of experimental coincidences as a function of the time window (black points) is compared to the expected number of accidental coincidences (red line), which was estimated scaling the number of coincidences observed in $\pm 1 \mathrm{~s}$ by a factor equal to the given coincidence time window.

For time windows larger than $1 \mathrm{~ms}$, the experimental coincidences are compatible, within the statistical uncertainties, with the expected spurious events. When smaller time windows are selected, an excess of coincidences is observed: the maximum signal significance was obtained for $\Delta T=6.4 \times 10^{-5} \mathrm{~s}$, at which we measured 48 events, against 29 accidental coincidences. An excess of $19 \pm 9$ events is abundantly higher than statistical fluctuations, being the corresponding $p$-value of the order of $10^{-3}$.

A more in-depth study of the characteristics of these 48 events shows that they seem to be randomly distributed in terms of time of occurrence and distance of sites involved. Moreover, since we have access to the direction cosines of the tracks reconstructed in each telescope, it is possible to check if the candidate events are correlated in orientation as well as in time. Assuming that the average direction of the tracks in a multi-track event is a good approximation of the EAS axis, we studied the distribution of the relative angle between the axes of the coincident EASs. From a first evaluation, the relative angles obtained for the candidate events do not significantly differ from those obtained for accidental coincidences. It is worth mentioning that such result can be affected by the finite angular resolution of the EEE telescopes in the reconstruction of the tracks $\left(\sim 1^{\circ}\right)$ and by the approximation done estimating the EAS axis as the average direction of the tracks.

Additional investigations are still ongoing, aimed at understanding the origin and characteristics of multi-track events and to study the features of the candidates events, exploiting all the information provided by the EEE telescopes.

The results discussed in this paper suggest a promising hint of the first detection of time correlated EASs. The characteristics of the EEE array and its performance, together with the huge amount 


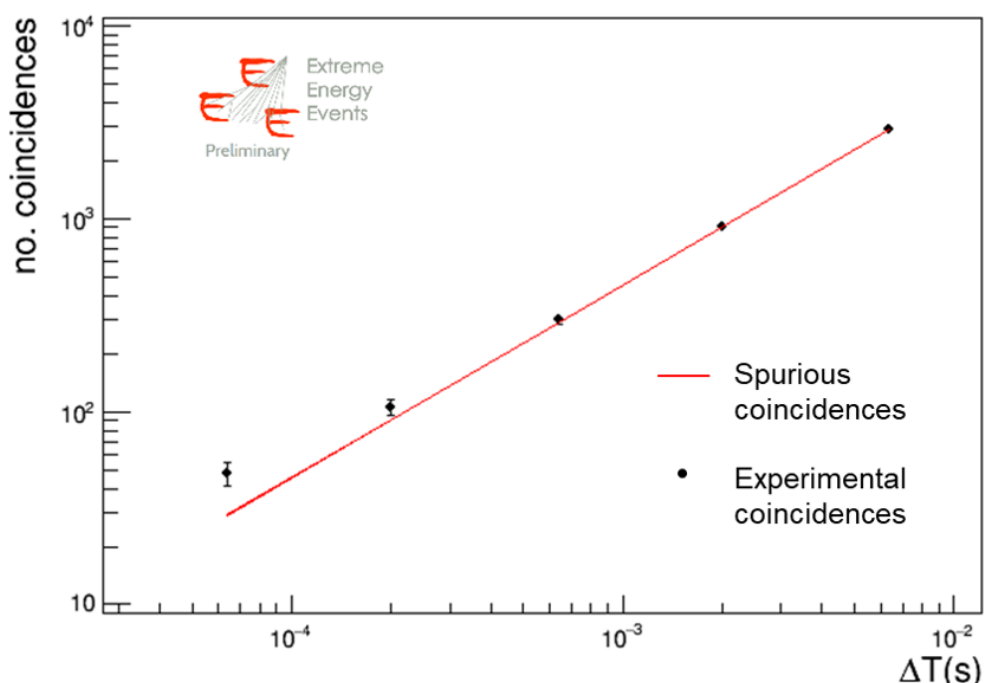

Figure 4: Number of coincident events (in black) as a function of the time window, compared with the expected number of accidental coincidences (red line). The cuts d $>5 \mathrm{~km}$ and $N_{\text {tracks }}>3$ were applied.

of data already available, allows to conclude that the EEE array is an ideal system to perform the search of rare long-range EAS coincidences.

\section{References}

[1] N. Gerasimova, G. Zatsepin. Disintegration of cosmic ray nuclei by solar photons. Sov. Phys. JETP 11 (1960) 899.

[2] J. Puget, F. Stecker, J. Bredekamp. Photonuclear interactions of ultrahigh energy cosmic rays and their astrophysical consequences. Astrophys. J. 205 (1976) 638.

[3] G. Medina-Tanco, A. Watson. The photondisintegration of cosmic ray nuclei by solar photons: The Gerasimova-Zatsepin effect revisited. Astropart. Phys. 10 (1999) 157.

[4] S. Mollerach, L. Epele, E. Roulet. On the disintegration of cosmic ray nuclei by solar photons. JHEP 03 (1999) 017.

[5] F. Stecker, M. Salamon. Photodisintegration of Ultrahigh Energy Cosmic Rays: A New Determination. Astrophys. J. 512 (1999) 521.

[6] G. Imponente, G. Sartorelli. Production mechanisms of multiple primaries for Cosmic Rays Showers. 29th ICRC 2005, arXiv:astro-ph/0607044v1 (2006).

[7] P. L. Rocca, F. Riggi. Nuclear Photodisintegration in the Solar Field: Numerical Simulations of the Gerasimova-Zatsepin Effect. Report INFN/AE-08/01, https://doi.org/10.15161/oar.it/1448969588.25 (2008).

[8] S. Lafebre, et al. Prospects for direct cosmic ray mass measurements through the GerasimovaZatsepin effect. Astron. Astrophys. 485 (2008) 1. 
[9] K. Andersen, S. Klein. High energy cosmic-ray interactions with particles from the sun. Phys. Rev. D 83 (2011) 103519.

[10] J. van Eijden, et al. Cosmic ray interactions in the solar system: The Gerasimova-Zatsepin effect. arXiv:astro-ph/1606.07693v2.

[11] N. Ochi, et al. Search for large-scale coincidences in network observation of cosmic ray air showers. J. Phys. G: Nucl. Part. Phys. 29 (2003) 1169.

[12] Y. Fujiwara, et al. Search for Simultaneous Parallel EAS Events in Long Baseline EAS Arrays with LAAS. Nucl. Phys. B (Proc. Suppl.) 151 (2006) 481.

[13] A. Iyono, et al. Cosmic ray composition studies through the Gerasimova-Zatsepin effects of heavy nuclei at LAAS. Astrophys. Space Sci. Trans. 7 (2011) 327.

[14] K. Smolek, et al. ALTA/CZELTA - a sparse very large air shower array: overview of the experiment and first results. 31st ICRC 2009, http://icrc2009.uni.lodz.pl/proc/pdf/icrc1300.pdf (2009).

[15] P. Blaschke, et al. CZELTA: An overview of the CZECH large area time coincidence array. Astrophys. Space Sci. Trans. 7 (2011) 69.

[16] http://eee.centrofermi.it

[17] M. Abbrescia et al. The Extreme Energy Events experiment: an overview of the telescopes performance. JINST 13 (2018) P08026 [arXiv:1805.04177].

[18] D. De Gruttola et al. Performance of the Multigap Resistive Plate Chambers of the Extreme Energy Events Project. JINST 14 (2019) C05022.

[19] M. Abbrescia et al. Observation of the february 2011 forbush decrease by the EEE telescopes. Eur. Phys. J. Plus 126 (2011) 61.

[20] M. Abbrescia et al. The EEE experiment project: status and first physics results. Eur. Phys. J. Plus 128 (2013) 62.

[21] M. Abbrescia et al. Looking at the sub-TeV sky with cosmic muons detected in the EEE MRPC telescopes. Eur. Phys. J. Plus 130 (2015) 187.

[22] M. Abbrescia et al. First detection of extensive air showers with the EEE experiment. Nuovo Cim. B 125 (2010) 243.

[23] S. Grazzi et al. EEE Project - Students from all parts of peninsula collaborate to study cosmic rays. Proceedings of Science, Volume 314, Poster PoS (EPS-HEP2017) 823

[24] M. Abbrescia et al. Search for long distance correlations between extensive air showers detected by the EEE network. Eur. Phys. J. Plus 133 (2018) 34.

[25] F. Riggi, et al. Time and orientation long-distance correlations between extensive air showers detected by the MRPC telescopes of the EEE project. Il Nuovo Cimento C 40 (2017) 196. 


\section{Full Authors List: EEE Collaboration}

M. Abbrescia ${ }^{1,2}$, C. Avanzini ${ }^{3}$, L. Baldini ${ }^{3,4}$, R. Baldini Ferroli ${ }^{5}$, G. Batignani ${ }^{3,4}$, M. Battaglieri ${ }^{6,7}$, S. Boi ${ }^{8,9}$, E. Bossini ${ }^{3,4}$, F. Carnesecchi ${ }^{10,11}$, C. Cicalò ${ }^{9}$, L. Cifarelli ${ }^{10,11}$, F. Coccetti ${ }^{12}$, E. Coccia ${ }^{13}$, A. Corvaglia ${ }^{14}$, D. De Gruttola ${ }^{15,16}$, S. De Pasquale ${ }^{15,16}$, F. Fabbri ${ }^{5}$, L. Galante ${ }^{17,18}$, M. Garbini ${ }^{10,12}$, G. Gemme ${ }^{6}$, I. Gnesi ${ }^{12,19}$, S. Grazzi ${ }^{6,20}$, D. Hatzifotiadou ${ }^{10,21}$, P. La Rocca ${ }^{22,23}$ Z. $\mathrm{Liu}^{24}$, G. Mandaglio ${ }^{20,23}$, G. Maron ${ }^{25}$, M. N. Mazziotta ${ }^{2}$, A. Mulliri ${ }^{8,9}$, R. Nania ${ }^{10}$, F. Noferini ${ }^{10}$, F. Nozzoli ${ }^{26}$, F. Palmonari ${ }^{10,11}$, M. Panareo ${ }^{14,27}$, M. P. Panetta ${ }^{12,14}$, R. Paoletti ${ }^{3,28}$, C. Pellegrino ${ }^{25}$, O. Pinazza ${ }^{10}$, C. Pinto ${ }^{22,23}$, S. Pisano ${ }^{5,12}$, F. Riggi ${ }^{22,23}$, G. C. Righini ${ }^{29}$, C. Ripoli ${ }^{15,16}$, M. Rizzi ${ }^{2}$, G. Sartorelli ${ }^{10,11}$, E. Scapparone ${ }^{10}$, M. Schioppa ${ }^{19,30}$, A. Scribano ${ }^{28}$, M. Selvi ${ }^{10}$, G. Serri ${ }^{8,9}$,

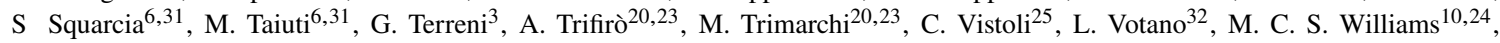
A. Zichichi ${ }^{10,11,21}$ and R. Zuyeuski ${ }^{24}$

${ }^{1}$ Dipartimento Interateneo di Fisica, Università di Bari, Bari, Italy. ${ }^{2}$ INFN Sezione di Bari, Bari, Italy. ${ }^{3}$ INFN Sezione di Pisa, Pisa, Italy. ${ }^{4}$ Dipartimento di Fisica, Università di Pisa, Pisa, Italy. ${ }^{5}$ INFN Laboratori Nazionali di Frascati, Frascati (Rome), Italy. ${ }^{6}$ INFN Sezione di Genova, Genova, Italy. ${ }^{7}$ Thomas Jefferson National Accelerator Facility, Newport News, VA, USA. ${ }^{8}$ Dipartimento di Fisica, Università di Cagliari, Cagliari, Italy. ${ }^{9}$ INFN Sezione di Cagliari, Cagliari, Italy. ${ }^{10}$ INFN Sezione di Bologna, Bologna, Italy. ${ }^{11}$ Dipartimento di Fisica ed Astronomia, Università di Bologna, Bologna, Italy. ${ }^{12}$ Museo Storico della Fisica e Centro Studi e Ricerche "E. Fermi", Rome, Italy. ${ }^{13}$ Gran Sasso Science Institute, LAquila, Italy. ${ }^{14}$ INFN Sezione di Lecce, Lecce, Italy. ${ }^{15}$ Dipartimento di Fisica, Università di Salerno, Salerno, Italy. ${ }^{16}$ INFN Gruppo Collegato di Salerno, Salerno, Italy. ${ }^{17}$ Dipartimento di Scienze Applicate e Tecnologia, Politecnico di Torino, Torino, Italy. ${ }^{18}$ INFN Sezione di Torino, Torino, Italy. ${ }^{19}$ INFN Gruppo Collegato di Cosenza, Cosenza, Italy. ${ }^{20}$ Dipartimento di Scienze Matematiche e Informatiche, Scienze Fisiche e Scienze della Terra, Università di Messina, Messina, Italy. ${ }^{21} \mathrm{CERN}$, Geneva, Switzerland. ${ }^{22}$ Dipartimento di Fisica e Astronomia, Università di Catania, Catania, Italy. ${ }^{23}$ INFN Sezione di Catania, Catania, Italy. ${ }^{24}$ ICSC World laboratory, Geneva, Switzerland. ${ }^{25}$ INFN-CNAF, Bologna, Italy. ${ }^{26}$ INFN Trento Institute for Fundamental Physics and Applications, Trento, Italy. ${ }^{27}$ Dipartimento di Matematica e Fisica, Università del Salento, Lecce, Italy. ${ }^{28}$ Dipartimento di Scienze Fisiche, della Terra e dell'Ambiente, Università di Siena, Siena, Italy. ${ }^{29}$ CNR Istituto di Fisica Applicata "Nello Carrara", Sesto Fiorentino (Florence), Italy. ${ }^{30}$ Dipartimento di Fisica, Università della Calabria, Rende (Cosenza), Italy. ${ }^{31}$ Dipartimento di Fisica, Università di Genova, Genova, Italy. ${ }^{32}$ INFN, Laboratori Nazionali del Gran Sasso, Assergi (L'Aquila), Italy. 